Chin. J. Astron. Astrophys. Vol. 0, No. 0, (200x) 000-000

Chinese Journal of

Astronomy and

Astrophysics

\title{
UBVI Surface Photometry of the Spiral Galaxy NGC 300 in the Sculptor Group
}

\author{
Sang Chul Kim ${ }^{1 \star}$, Hwankyung Sung ${ }^{2}$, Hong Soo Park ${ }^{3}$, and Eon-Chang Sung1 \\ ${ }^{1}$ Korea Astronomy Observatory, Taejon 305-348, Republic of Korea \\ ${ }^{2}$ Department of Astronomy and Space Science, Sejong University, 98 Gunja-dong, Gwangjin-gu, Seoul \\ 143-747, Republic of Korea \\ ${ }^{3}$ Astronomy Program, SEES, Seoul National University, Seoul 151-742, Republic of Korea
}

Received 2004 ; accepted 2004

\begin{abstract}
We present $U B V I$ surface photometry for $20 .^{\prime} 5 \times 20 .^{\prime} 5$ area of a late-type spiral galaxy NGC 300. In order to understand the morphological properties and luminosity distribution characteristics of NGC 300, we have derived isophotal maps, surface brightness profiles, ellipticity profiles, position angle profiles, and color profiles. By merging the $I$-band data of our surface brightness measurements with those of Böker et al. (2002) based on Hubble Space Telescope observations, we have made combined $I$-band surface brightness profiles for the region of $0 . .^{\prime \prime} 02<r<500^{\prime \prime}$ and decomposed the profiles into three components: a nucleus, a bulge, and an exponential disk.
\end{abstract}

Key words: galaxies: spiral — galaxies: photometry — galaxies: individual (NGC 300) — galaxies: nuclei

\section{INTRODUCTION}

NGC 300 (=ESO 295-G020, IRAS 00525-3757, PGC 3238) is a late-type (SA(s)d, de Vaucouleurs et al. 1991) spiral galaxy in the nearest galaxy group, the Sculptor group, which contains five major spiral galaxies (NGC 55, NGC 247, NGC 253, NGC 300, and NGC 7793) and 20 dwarf galaxies (Côté et al. 1997, Whiting 1999, Karachentsev et al. 2003; cf. van den Bergh 1999). NGC 300 is a rather bright $\left(M_{B}=-18.6\right)$ and nearly face-on galaxy, similar to M33 in the northern hemisphere (Blair \& Long 1997). Some basic information of this galaxy is summarized in Table 1, which is supplemented to the table 1 of Kim, Sung, \& Lee (2002, hereafter Paper I).

Although there have been several studies on various objects in NGC 300 (e.g. Paper I and references therein), only a few studies exist on surface photometry study on this galaxy. De Vaucouleurs \& Page (1962)

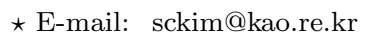


Table 1 Basic Information of NGC 300

\begin{tabular}{|c|c|c|}
\hline Parameter & Information & Reference \\
\hline$\alpha_{J 2000}, \delta_{J 2000}$ & $0^{h} 54^{m} 53 .^{s} 48,-37^{\circ} 41^{\prime} 03 .^{\prime \prime} 8$ & NASA/IPAC Extragalactic Database \\
\hline$l, b$ & $299 .^{\circ} 21,-79 .^{\circ} 42$ & NASA/IPAC Extragalactic Database \\
\hline Type & $\mathrm{SA}(\mathrm{s}) \mathrm{d}$ & de Vaucouleurs et al. 1991 \\
\hline H I heliocentric radial velocity, $v_{\odot}$ & $142 \pm 4 \mathrm{~km} \mathrm{~s}^{-1}$ & de Vaucouleurs et al. 1991 \\
\hline Maximum rotational velocity, $V_{\max }$ & $87 \mathrm{~km} \mathrm{~s}^{-1}$ & Carignan 1985 \\
\hline Foreground reddening, $E(B-V)$ & $0.013 \mathrm{mag}$ & Schlegel et al. 1998 \\
\hline Isophotal major diameter at the $25.0 \mathrm{~B} \mathrm{mag} \operatorname{arcsec}^{-2}$ level, $\mathrm{D}_{25}$ & $21 .^{\prime} 9$ & de Vaucouleurs et al. 1991 \\
\hline Position angle & $111^{\circ}$ & de Vaucouleurs et al. 1991 \\
\hline Minor to major axis ratio at $\mathrm{D}_{25},(b / a)_{25}$ & 0.74 & Carignan 1985 \\
\hline Inclination $i$ & $42 .^{\circ} 3 \pm 3 .^{\circ} 0$ & Carignan 1985 \\
\hline Distance modulus, $(\mathrm{m}-\mathrm{M})_{0}$ & $26.53 \pm 0.07 \mathrm{mag}$ & $\begin{array}{l}\text { Freedman et al. } 2001 \\
\text { (cf. Butler et al. 2004) }\end{array}$ \\
\hline Distance, $d$ & $2.02 \pm 0.07 \mathrm{Mpc}\left(1^{\prime \prime}=9.8 \mathrm{pc}\right)$ & Freedman et al. 2001 \\
\hline Exponential disk corrected central surface brightness, $\mu_{B}(0)$ & $22.23 \mathrm{mag} \operatorname{arcsec}^{-2}$ & Carignan 1985 \\
\hline Exponential disk corrected central surface brightness, $\mu_{I}(0)$ & $19.97 \mathrm{mag} \operatorname{arcsec}^{-2}$ & This study \\
\hline Scale length, $r_{s}$ (obtained from $B$-band) & $2.06 \mathrm{kpc}$ & Carignan 1985 \\
\hline Scale length, $r_{s}$ (obtained from $I$-band) & $1.47 \mathrm{kpc}$ & This study \\
\hline Apparent total magnitude, $B_{\mathrm{T}}$ & $8.72 \pm 0.05 \mathrm{mag}$ & de Vaucouleurs et al. 1991 \\
\hline Apparent total color index, $(B-V)_{\mathrm{T}}$ & $0.59 \pm 0.03 \mathrm{mag}$ & de Vaucouleurs et al. 1991 \\
\hline Apparent total color index, $(U-B)_{\mathrm{T}}$ & $0.11 \pm 0.03 \mathrm{mag}$ & de Vaucouleurs et al. 1991 \\
\hline Corrected total magnitude, $B_{\mathrm{T}}^{0}$ & 8.53 & de Vaucouleurs et al. 1991 \\
\hline Corrected total color index, $(B-V)_{\mathrm{T}}^{0}$ & 0.56 & de Vaucouleurs et al. 1991 \\
\hline Corrected total color index, $(U-B)_{\mathrm{T}}^{0}$ & 0.09 & de Vaucouleurs et al. 1991 \\
\hline Absolute total magnitude, $M_{B_{T}}$ & -18.59 & Sandage \& Tammann 1981 \\
\hline $\begin{array}{l}\text { Metallicity from the ratio of carbon stars to } \mathrm{M} \text { stars of } \\
\text { spectral type M5 or later, }[\mathrm{Fe} / \mathrm{H}]\end{array}$ & -0.5 dex & Richer, Pritchet, \& Crabtree 1985 \\
\hline $\mathrm{H}$ I column density in the direction of NGC 300 & $2.97 \times 10^{20}$ atoms $\mathrm{cm}^{-2}$ & Read, Ponman, \& Strickland 1997 \\
\hline H I flux & $\leq 670 \mathrm{Jy} \mathrm{km} \mathrm{s}{ }^{-1}$ & Carignan 1985 \\
\hline H I mass, $M_{H I}$ & $\overline{(2.5} \pm 0.1) \times 10^{9} M_{\odot}$ & Rogstad, Crutcher, \& Chu 1979 \\
\hline
\end{tabular}

performed surface photometry study of NGC 300 using photographic plates obtained at Mount Stromlo 30- and 74-inch reflectors. Carignan (1985) has performed surface photometry of the three Sculptor group galaxies: NGC 7793, NGC 247, and NGC 300. He has used $B_{J}$-band photographic plates taken by using the 1.2m Schmidt telescope at Siding Spring Observatory. Recently, Böker et al. (2002, 2004) have observed 77 nearby late-type spiral galaxies including NGC 300 using the Hubble Space Telescope (HST)/WFPC2. They have used the $I$-band images of the galaxies to study the nuclear star clusters and identified distinct, compact, and dominant sources at or very close to the photocenter of the 59 galaxies of their sample, which include NGC 300. They have presented the $I$-band surface brightness profiles of NGC 300 inside of $r \sim 15^{\prime \prime}$ and some photometric parameters of the nuclear star cluster in NGC 300.

There have not been any surface photometry study on NGC 300 with multi-band filters and CCD images. In this paper, we present surface photometry based on $U B V I$ CCD images for the $20 .^{\prime} 5 \times 20 .^{\prime} 5$ areas of NGC 300. We have derived surface brightness distributions, color distributions, and structural parameters for $r<500^{\prime \prime}$ of NGC 300. This paper is composed as follows: Section 2 describes the photometric observations and the data reduction process. The isophotal maps of NGC 300 are presented in Section 3. In Section 4 ellipse fitting results are presented, and color profiles are presented in Section 5. In Section 6 we compare the surface photometry results with those of previous studies and present the surface brightness profile decomposition results. Finally, a brief summary is given in Section 7 . 


\section{OBSERVATIONS AND DATA REDUCTION}

$U B V I$ CCD photometry was performed on 1997 November 23 at Siding Spring Observatory with the 40 inch $(1 \mathrm{~m})$ telescope $(\mathrm{f} / 8)$ and a thinned SITe $2048 \times 2048 \mathrm{CCD}\left(24 \mu \mathrm{m}\right.$ pixels). The scale was $0 .^{\prime \prime} 602$ pixel $^{-1}$, giving 20.'5 on a side. Exposure times used in the observations were $1200 \mathrm{~s}$ in $U, 600 \mathrm{~s}$ in $B, 300 \mathrm{~s}$ in $V$, and $120 \mathrm{~s}$ in $I$. The night was photometric and the seeings were $1 .^{\prime \prime} 5,1 .^{\prime \prime} 6,1 . .^{\prime \prime} 4$, and 1. " $^{\prime} 4$ in the $U, B, V$, and $I$ images, respectively.

All the preprocessing, such as overscan correction, bias subtraction and flat fielding were done using the IRAF*/CCDRED package. Surface photometry of NGC 300 from the CCD images have been performed by using the ellipse fitting task IRAF/SPIRAL (Surface Photometry Interactive Reduction and Analysis Library), which is an ellipse fitting package developed at Kiso observatory for galaxy surface photometry (Ichikawa et al. 1987) and is essentially the same as that described by Kent (1983). Smoothing of the images have been done using variable-width Gaussian beam of IRAF/SPIRAL.SMOOTH task to improve the signal-to-noise ratio in the outer regions of the galaxy.

The instrumental magnitudes were transformed to the standard Johnson-Cousins $U B V I$ system using the observation data of equatorial standards of South African Astronomical Observatory (SAAO) (Menzies et al. 1991) and of blue and red standards by Kilkenny et al. (1998), both of which were observed on the same night. The atmospheric extinction coefficients were determined from the photometry of standard stars and the transformation coefficients adopted by Sung \& Bessell (2000) were used. The transformation equations we derived from the photometry of the standard stars are $u=U+4.987+0.545 \times X_{u}+u 3 \times(U-B)$, $b=B+2.542+0.289 \times X_{b}+0.101 \times(B-V), v=V+2.412+0.147 \times X_{v}-0.072 \times(B-V)$, and $i=I+2.869+0.090 \times X_{i}-0.028 \times(V-I)$, where lowercase and uppercase letters represent, respectively, the instrumental system and standard system (zeropoint= 25.0). $X$ represents the air mass and u3 is -0.006 when $(U-B) \geq 0.0$ and -0.125 when $(U-B)<0.0$. The rms scatter of the standard stars was 0.012 mag for $U, 0.008 \mathrm{mag}$ for $B, 0.011 \mathrm{mag}$ for $V$, and $0.015 \mathrm{mag}$ for $I$, indicating the night was of good photometric quality.

The sky background values were determined from the mode values of the south-east edges of the images which are the farthest and clear regions from the galaxy (the distance from the center of NGC 300 is $\left.\sim 800^{\prime \prime}\right)$. These sky background values were then subtracted from the individual images. We have calculated the surface brightnesses for these sky values, obtaining $21.59 \pm 0.22,22.33 \pm 0.08,21.58 \pm 0.07$, and $19.04 \pm 0.03$ mag $\operatorname{arcsec}^{-2}$ for $U, B, V$, and $I$ band, respectively. The errors quoted here are the estimated standard deviations of background sky brightnesses. Considering that the lunar phase when our data were obtained is $23 \mathrm{~d}$ after new Moon (or $7 \mathrm{~d}$ before the next new Moon) and that the airmass of our target is in the range of $1.00-1.02$, our sky brightness estimates seem to be reasonably similar to those of the Kitt Peak National Observatory (Elias 1994; Kim, Lee, \& Geisler 2000) or those of Crimea/Hawaii sites (Leinert et al. 1995).

*IRAF is distributed by the National Optical Astronomy Observatories, which are operated by the Association of Universities for Research in Astronomy, Inc., under cooperative agreement with the National Science Foundation. 

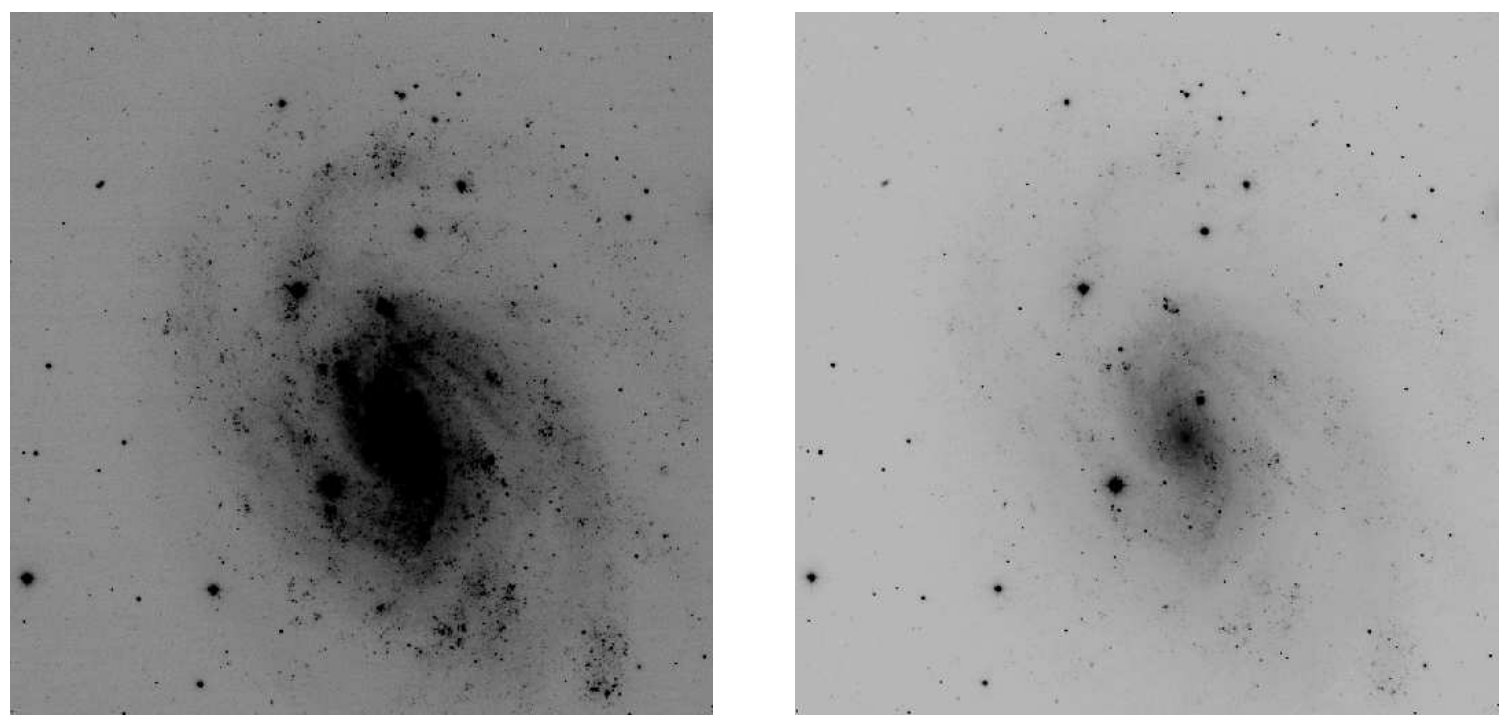

Figure 1 CCD images of NGC 300. North is to the right of the image and east is at the top. The scale is $0 .^{\prime \prime} 602$ pixel $^{-1}$ and the size of the field is $20 .^{\prime} 5 \times 20 .^{\prime} 5$. (a: left panel) A greyscale map of the $B$ CCD image of NGC 300. The spiral arm features are easily seen in this figure. (b: right panel) A greyscale map of the $V$ CCD image of NGC 300. This figure shows the central region of the galaxy with faint features of the spiral arms. Most of the brightest stars are foreground stars in both of the figures.

\section{MORPHOLOGICAL CHARACTERISTICS OF NGC 300}

Greyscale maps of $B$ and $V$ CCD images of NGC 300 are shown in Fig. 1 (a) and (b), respectively. The size of the images is $20.5 \times 20 .^{\prime} 5$. Most of the brightest stars in the images are foreground stars. The $B$-band image in Fig. 1 (a) shows the spiral features well, which manifests several lumps of bright stars that are stellar associations (Pietrzyński et al. 2001; Paper I). The central region of NGC 300 shown in Fig. 1 (b) reveals that the bulge of this $\mathrm{SA}(\mathrm{s}) \mathrm{d}$ type galaxy is quite small compared to the size of the spiral arms, which will be discussed in Section 6.2 .

Fig. 2 shows the isophotal maps of NGC 300. Here the X and $\mathrm{Y}$ axes are in pixel units and one tick interval corresponds to $60 .{ }^{\prime \prime}$. The contour interval is $0.5 \mathrm{mag} \operatorname{arcsec}^{-2}$. North is to the right of the image and east is at the top. As can be seen in Fig. 2, there is no conspicuous bulge isophotes and most isophotes are elongated NW-SE direction excluding the innermost one. Even though the eastern spiral arm is clearly seen in Fig. 2 (a) and (b), the (north-)western arm is barely delineated thanks to the large association of bright stars at around $(\mathrm{X}=1750, \mathrm{Y}=100)$. These two major arms are those noted by Sandage \& Bedke (1994).

\section{ELLIPSE FITTING}

We have applied the ellipse fitting task IRAF/SPIRAL.PROFS.VPROF to the NGC 300 images to obtain surface brightness profiles as well as profiles of ellipticity $(\epsilon)$ and position angle (PA). 

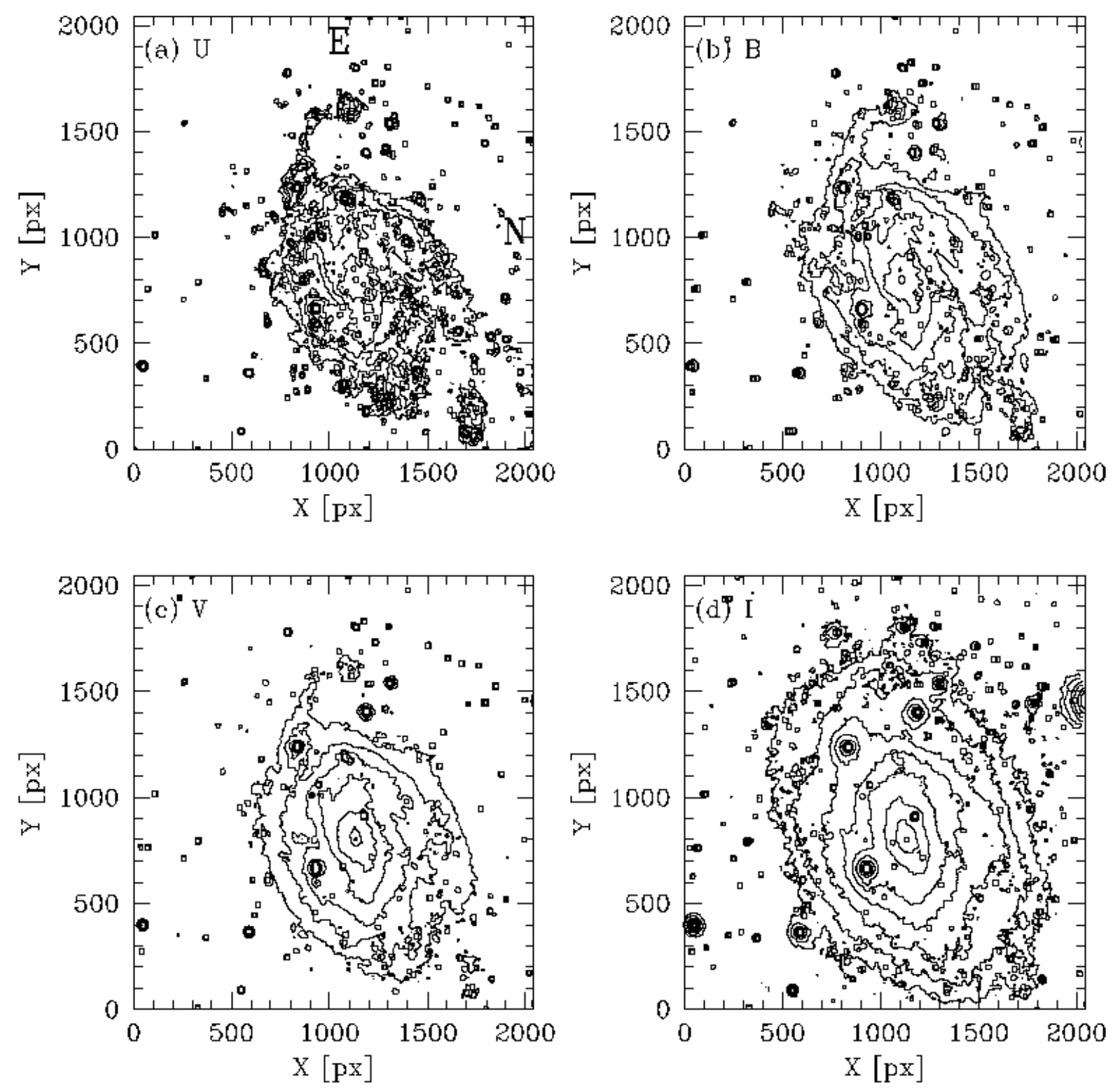

Figure 2 Isophotal contour maps of NGC 300 in (a) $U$-band, (b) $B$-band, (c) $V$-band, and (d) $I$-band. North is to the right of the image and east is at the top. The size of each field is $20 .^{\prime} 5 \times 20 .^{\prime} 5$ and one tick interval corresponds to $60 .^{\prime \prime} 2$. The contour interval is $0.5 \mathrm{mag} \operatorname{arcsec}^{-2}$. 
Fig. 3 shows $U, B, V$, and $I$ radial surface brightness profiles of NGC 300 along the major axis for the region of $r<500^{\prime \prime}$. The typical errors of $B$ and $V$-band surface brightnesses are denoted at the lower and upper panels, respectively. Since the IRAF/SPIRAL task does not give the error of the surface brightnesses, we have used the IRAF/STSDAS.ANALYSIS.ISOPHOTE.ELLIPSE task at the given radius using the ellipticity, position angle, and center coordinates obtained by SPIRAL task just to obtain the brightness errors. Unlike the elliptical galaxies which have smooth brightness profiles, spiral galaxies are patchy and have spiral arm features. The local patchy features lead to non-systematic error values as in Fig. 3, though the low photon signals relative to the sky brightness make the errors get increased at the outer radii.

The surface brightness profiles in $U$ and $B$ are very similar in their values, which can be deduced from the apparent total $(U-B)$ color index of NGC 300 of $0.11 \pm 0.03$ mag (de Vaucouleurs et al. 1991). While the $U$ surface brightnesses are fainter than those of $B$-band at $r<200^{\prime \prime}$, they get brighter at $r>200^{\prime \prime}$.

Fig. 4 shows ellipticity $(\epsilon=1-b / a$, where $a$ and $b$ are semi-major and semi-minor axis lengths, respectively) profiles of NGC 300 in $U, B, V$, and $I$-bands. The straight lines are plotted based on eye-fitting and are the same for all bands. Ellipticity increases from $\sim 0$ (at $r \sim 2^{\prime \prime}$ ) to $\sim 0.5$ (at $r \sim 200^{\prime \prime}$ ), and then decreases outward. The amplitude of ellipticity variation is rather larger in $U$ - and $B$-bands than in the $V$ and $I$-bands, and the absolute values of ellipticities at $r>100^{\prime \prime}$ in $U$ - and $B$-bands are also larger than those in $V$ - and $I$-bands.

Fig. 5 shows position angle (PA; $\mathrm{N}$ through $\mathrm{E}$ to the major axis) profiles of NGC 300 in $U, B, V$, and I-bands. The straight lines are plotted based on eye-fitting and are the same for all bands. Position angles increase from $\sim 40^{\circ}$ (at $r \sim 1^{\prime \prime}$ to $2^{\prime \prime}$ ) to $\sim 110^{\circ}$ (at $r \sim 40^{\prime \prime}$ ), and fluctuate around the value of $\sim 110^{\circ}$ at regions of $r>40^{\prime \prime}$. This PA value of $\sim 110^{\circ}$ is in good agreement with those obtained by Carignan (1985; 105. $\left.{ }^{\circ} \pm 1 .{ }^{\circ} 8\right)$ and by de Vaucouleurs et al. $\left(1991 ; 111^{\circ}\right)$. The changes in position angles are mainly due to the spiral structure of NGC 300 and there is seen no region of flat PAs (which is an indicator of the bar structure; Choi et al. 1998).

\section{COLOR PROFILES}

Fig. 6 shows the radial surface color profiles of NGC 300 along the major axis. Here the surface color means the differential color per square arcsecond. The errors for $(B-V)$ color are ploted at the upper panel in order to show the typical error values. The solid lines are least-square fits to the data that yield the color gradient slopes of $-0.07 \mathrm{mag} \operatorname{arcsec}^{-2}\left(100^{\prime \prime}\right)^{-1}$ for $(U-B),-0.11 \mathrm{mag} \operatorname{arcsec}^{-2}\left(100^{\prime \prime}\right)^{-1}$ for $(U-V)$, $-0.14 \mathrm{mag} \operatorname{arcsec}^{-2}\left(100^{\prime \prime}\right)^{-1}$ for $(U-I),-0.04 \mathrm{mag} \operatorname{arcsec}^{-2}\left(100^{\prime \prime}\right)^{-1}$ for $(B-V),-0.07 \mathrm{mag} \operatorname{arcsec}^{-2}$ $\left(100^{\prime \prime}\right)^{-1}$ for $(B-I)$, and $-0.04 \mathrm{mag} \operatorname{arcsec}^{-2}\left(100^{\prime \prime}\right)^{-1}$ for $(V-I)$. As the wavelength separation between the two filters gets larger, the slope of the corresponding color profile gets steeper. Here, the steepest slope is for the $(U-I)$ color, and the least steep slopes are for the $(B-V)$ and $(V-I)$ colors.

NGC 300 shows typical color profiles of late-type spiral galaxies, being redder in the inner regions and getting bluer as the radius increases. The primary factor for this negative radial color gradient is the 


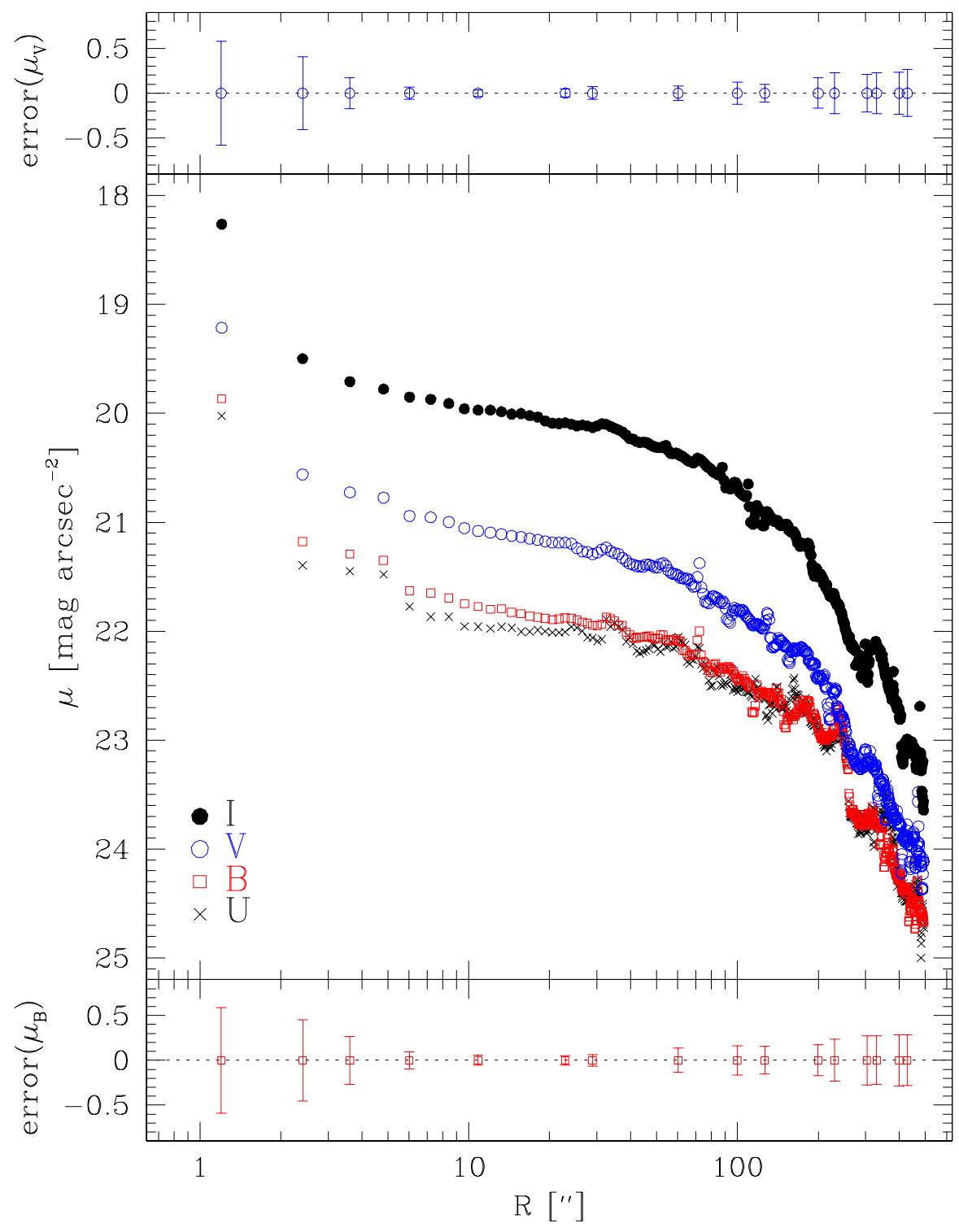

Figure $3 U, B, V$, and $I$ major axis surface brightness profiles of NGC 300 . The typical errors of $B$ and $V$-band surface brightnesses are denoted at the lower and upper panels, respectively. The data are available from one of the authors (S.C.K.). 

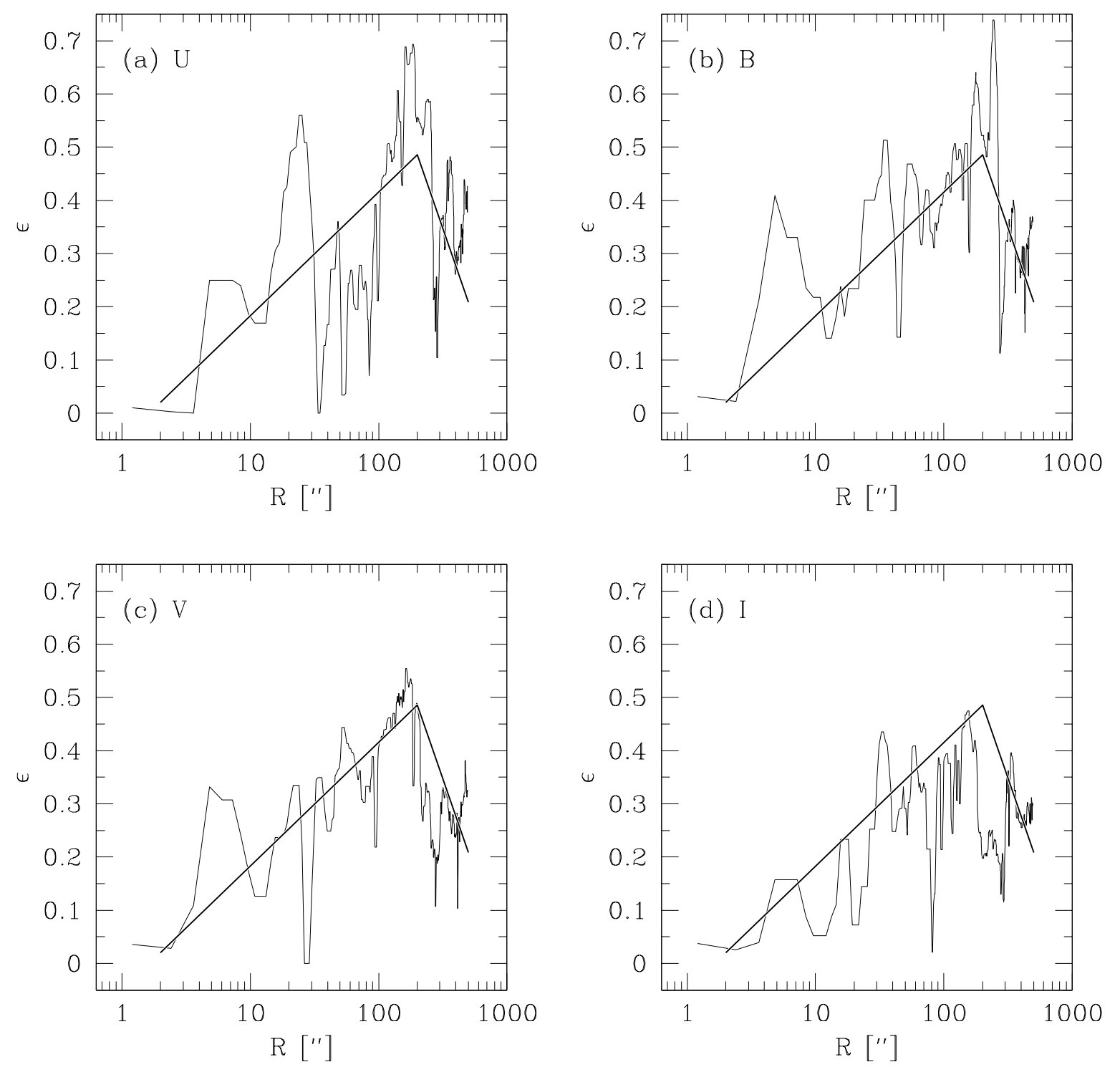

Figure 4 Ellipticity profiles of NGC 300. The straight lines are based on eye-fitting and are the same for all bands. 

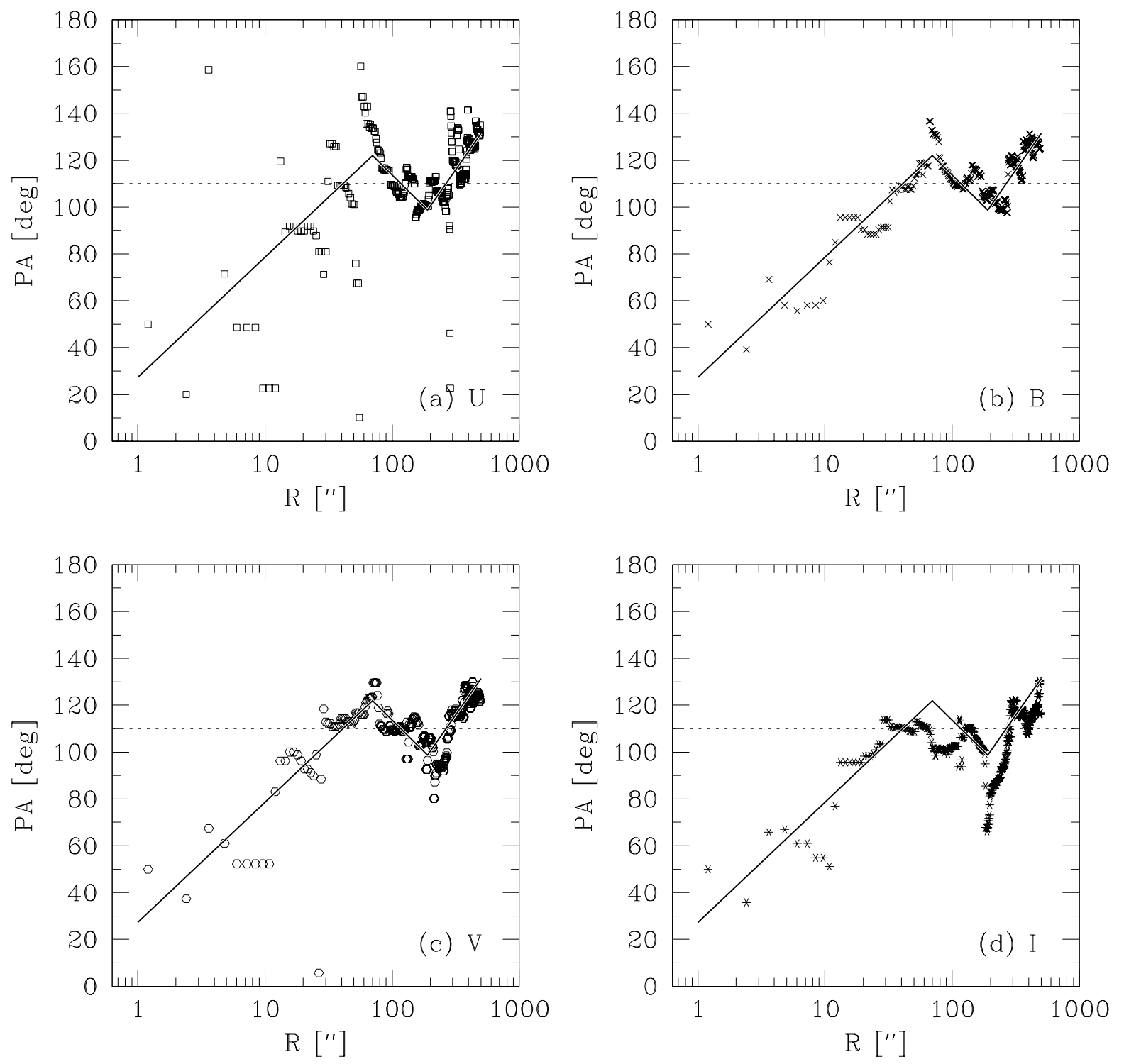

Figure 5 Position angle profiles of NGC 300. The straight lines are based on eye-fitting and are the same for all bands. 


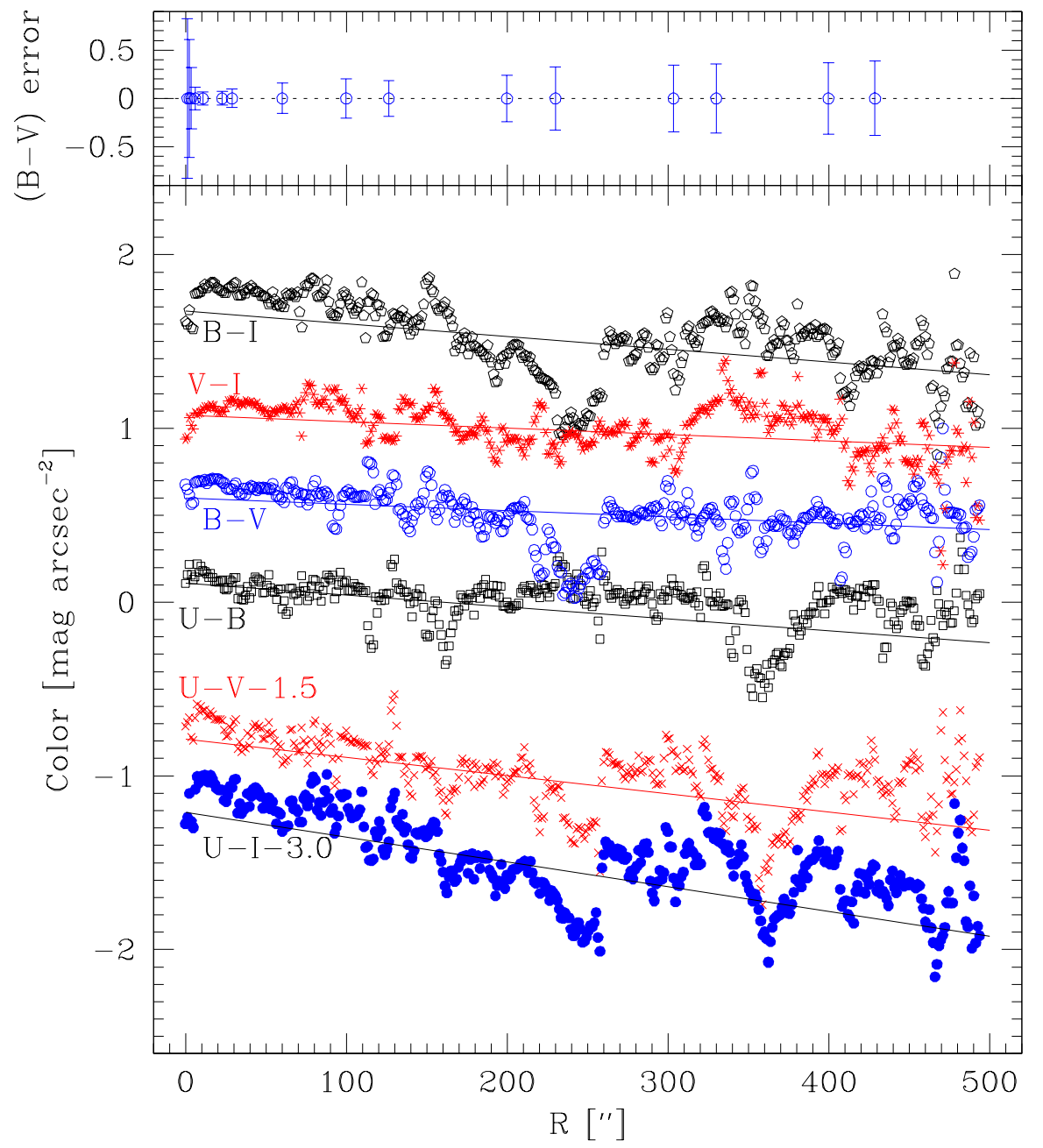

Figure 6 Radial surface color profiles of NGC 300. The errors for $(B-V)$ color are ploted at the upper panel to show the typical error values. 

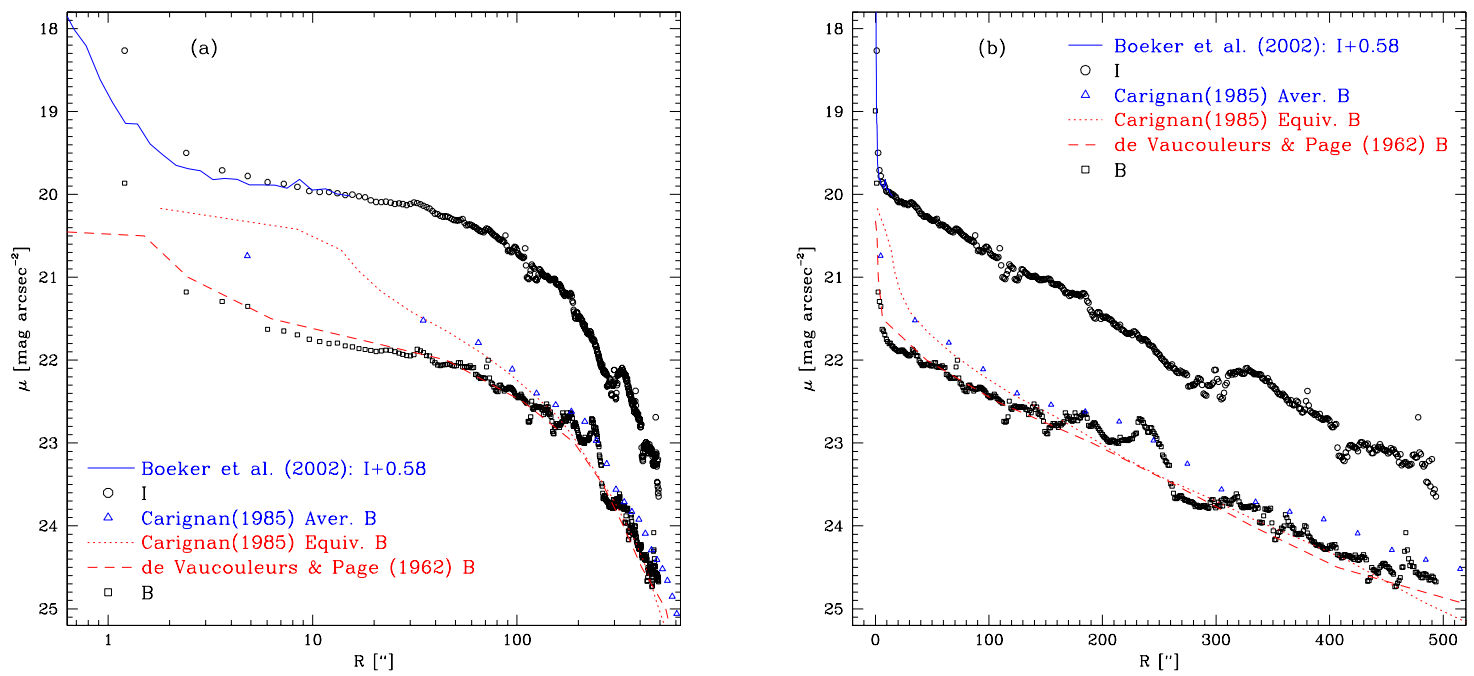

Figure 7 Comparison of the surface brightness profiles obtained in this study with those of previous studies in logarithmic radius scale (panel a) and linear radius scale (panel b). $B$ and $I$ surface brightnesses obtained in this study are denoted as open squares and open circles, respectively. $B_{J}$ magnitudes of Carignan (1985) are transformed to $B$ magnitudes using the relation $B=B_{J}+0.06\left(B_{J}-R_{F}\right)=B_{J}+0.05$ (Carignan 1985). $0.58 \mathrm{mag} \operatorname{arcsec}^{-2}$ is added to the Böker et al. (2002)'s $I$-band surface brightnesses to match their profiles to those obtained in this study.

differences of the underlying stellar populations at the inner and outer parts of the galaxies (Kim \& Ann 1990).

\section{DISCUSSION}

\subsection{Comparison with Previous Studies}

Fig. 7 shows the comparison of the surface brightness profiles obtained in this study with those of previous studies. Fig. 7 (a) is in logarithmic radius scale and Fig. 7 (b) is in linear radius scale. $B$ - and $I$-band surface brightness profiles obtained in this study are denoted as open squares and open circles, respectively. Both de Vaucouleurs \& Page (1962) and Carignan (1985) have given B-band surface brightness profiles obtained from photographic plates, which are denoted as dashed lines and dotted lines (equivalent profile) or open triangles (elliptically averaged profile), respectively. $B_{J}$ magnitudes of Carignan (1985) are transformed to $B$ magnitudes using the relation $B=B_{J}+0.06\left(B_{J}-R_{F}\right)=B_{J}+0.05$ (Carignan 1985). Recent HST-based $I$-band surface brightness profiles for the inner $r<15^{\prime \prime}$ region of NGC 300 given by Böker et al. (2002) are denoted by solid lines. Because the Böker et al. (2002)'s $I$-band surface brightnesses are $0.58 \operatorname{mag} \operatorname{arcsec}^{-2}$ brighter than those in this study, we have added $0.58 \mathrm{mag} \operatorname{arcsec}^{-2}$ to their values to match the two profiles.

For the $B$-band surface brightness profiles, our profiles are in good agreement with those of de Vaucouleurs \& Page (1962) at $r \lesssim 160^{\prime \prime}$ and with equivalent profiles of Carignan (1985) at the outer region. Carignan (1985) notes the differences between his and de Vaucouleurs \& Page (1962)'s surface brightness 
profiles, which is up to $0.4 \mathrm{mag} \operatorname{arcsec}^{-2}$ and is larger at the central region. This difference between the studies of de Vaucouleurs \& Page (1962) and Carignan (1985) seems to be due to some problems in the density-to-intensity calibration of the photographic plates since both studies are based on photographic plate photometry.

The $I$-band surface brightness profiles of NGC 300 given by Böker et al. (2002) are $\sim 0.6$ mag $\operatorname{arcsec}^{-2}$ brighter than those of our study. Since Böker et al. (2002) have observed their galaxies with the F814W filter only, they have made the photometric calibration and conversion to Johnson $I$-band assuming a standard color of $V-I=1$ for all the galaxies. Nevertheless, this does not account for the rather large difference between the two photometric studies, because even $1 \mathrm{mag}$ of change in the color of a galaxy gives only $\sim$ $0.01 \mathrm{mag}$ difference of zero point as they mentioned. Apart from the zeropoint difference between the two studies and some discrepancies of the values at a few central points, the two $I$-band surface brightnesses show reasonable agreement especially at $r>5^{\prime \prime}$. For the very central regions of $r<5^{\prime \prime}$, Böker et al. (2002)'s results based on the HST high-resolution CCD images deserve to be considered as reliable.

\subsection{Surface Brightness Profile Decomposition}

We have combined our $I$-band surface brightness measurements for the region of $r>5^{\prime \prime}$ with those of Böker et al. (2002) for the region from $0 .{ }^{\prime \prime} 02$ to $5^{\prime \prime}$. The resulting $I$-band surface brightness profile is shown in Fig. 8 as open circles. Using this combined surface brightness profile for the region of $0 .{ }^{\prime \prime} 02<r<500^{\prime \prime}$, we have performed a three component decomposition: a nucleus, a bulge and a disk (Stephens \& Frogel 2002). We assume an exponential disk

$$
I_{d i s k}(r)=I_{0} \exp \frac{-r}{R_{d}}
$$

with a central intensity $I_{0}$ and a disk scale length $R_{d}$, and a de Vaucouleurs $r^{1 / 4}$ bulge

$$
I_{\text {bulge }}(r)=I_{e} \exp \left\{-7.67\left[\left(\frac{a r}{R_{e}}\right)^{1 / 4}-1\right]\right\}
$$

with an effective radius $R_{e}$ and an intensity $I_{e}$ at $R_{e}$. The factor $a=\left[\left(1+\cos ^{2} i\right) / 2\right]^{1 / 2}$ is a correction factor for deprojecting a spherical bulge, and an inclination of $i=42 .{ }^{\circ} 3$ (Carignan 1985) is used. For the core component, we have used Sersic model (Sersic 1968):

$$
I_{\text {core }}(r)=I_{s} \exp \left\{-b_{n}\left[\left(\frac{a r}{R_{s}}\right)^{1 / n}-1\right]\right\}
$$

where $R_{s}$ is an effective radius, $I_{s}$ is an intensity at $R_{s}$, and $n$ is the shape index $\left(b_{n} \approx 1.9992 n-0.3271\right)$. The result of the three-component decomposition is shown in Fig. 8, and the parameter values are listed in Table 2. Fig. 8 shows that NGC 300 has three components: the central peak in surface brightness at $r<1^{\prime \prime}$ which is reasonably fitted by the Sersic function, the disk that has a central surface brightness of $19.97 \mathrm{mag} \operatorname{arcsec}^{-2}$ and scale length of $R_{d}=2.5$ (=1.47 kpc), and a very faint spheroidal component which is even fainter than that of M33 (Stephens \& Frogel 2002).

The nuclear star cluster observed by Böker et al. (2002) using the HST has been included in the globular cluster candidate catalogue in Paper I with highest probability. It would be helpful to perform a spectroscopic observation on this nuclear star cluster of NGC 300 to confirm the suggestion of Soffner et al. 
(1996) that the nucleus of this galaxy is an unresolved compact stellar cluster and to understand the nature of it. Information on the velocity dispersion of this object will also give a clue on the existence of any black hole in it (Gebhardt et al. 2001; Gebhardt, Rich, \& Ho 2002).

More surface brightness data in other passbands at high spatial resolution will be valuable to get information on the existence of any color gradient in the nuclear region of NGC 300 (Kormendy \& McClure 1993; Kim \& Lee 1998).

Table 2 Model Parameters for $I$-band Three-Component Decomposition

\begin{tabular}{c|lc}
\hline & Parameter & Value \\
\hline Disk & scale length, $R_{d}$ & $150^{\prime \prime}=2 . .^{\prime} 50=1.47 \mathrm{kpc}^{\mathrm{a}}$ \\
& central surface brightness, $\mu_{0}$ & $19.97 \mathrm{mag} \operatorname{arcsec}^{-2}$ \\
\hline Bulge & effective radius, $R_{e}$ & $1900^{\prime \prime}=31 .^{\prime} 67=18.62 \mathrm{kpc}^{\mathrm{a}}$ \\
& surface brightness at $R_{e}, \mu_{e}$ & $29.25 \mathrm{mag} \operatorname{arcsec}^{-2}$ \\
\hline Core & effective radius, $R_{s}$ & $0 .^{\prime \prime} 094=0.92 \mathrm{pc}^{\mathrm{a}}$ \\
& surface brightness at $R_{s}, \mu_{s}$ & $16.55 \mathrm{mag} \operatorname{arcsec}^{-2}$ \\
& shape index, $n$ & 1.43 \\
\hline
\end{tabular}

Note. ${ }^{a}$ Assuming the distance modulus of $(\mathrm{m}-\mathrm{M})_{0}=26.53 \pm 0.07$ mag (Freedman et al. 2001), which gives $1^{\prime \prime}=9.8 \mathrm{pc}$.

\section{SUMMARY}

We have presented surface photometry for the $20 .{ }^{\prime} 5 \times 20 .^{\prime} 5$ area of SA(s)d galaxy NGC 300 in the Sculptor group. We have derived the isophotal maps, surface brightness profiles, ellipticity profiles, position angle profiles, and color profiles. We have decomposed the $I$-band surface brightness profiles of $0 .{ }^{\prime \prime} 02<r<$ $500^{\prime \prime}$ region into a nucleus, a bulge, and an exponential disk.

\section{ACKNOWLEDGMENTS}

We thank the anonymous referee for the constructive comments and suggestions that have improved this paper. S.C.K. is grateful to Mr. Yoon-Ho Park and Mr. Chang Hyun Baek for providing information on the SPIRAL package, and to Dr. Bong Gyu Kim for his continuous and graceful encouragements on astronomical research. This research has made use of the NASA/IPAC Extragalactic Database (NED) which is operated by the Jet Propulsion Laboratory, California Institute of Technology, under contract with the National Aeronautics and Space Administration. H. S. acknowledges the support of the Korea Science and Engineering Foundation to the Astrophysical Research Center for the Structure and Evolution of the Cosmos (ARCSEC) at Sejong University. The data used in this paper are available by e-mail request to sckim@kao.re.kr.

\section{REFERENCES}

Blair W. P., Long K. S., 1997, ApJS, 108, 261

Böker T., Laine S., van der Marel R. P., Sarzi M., Rix H.-W., Ho L. C., Shields J. C., 2002, AJ, 123, 1389 


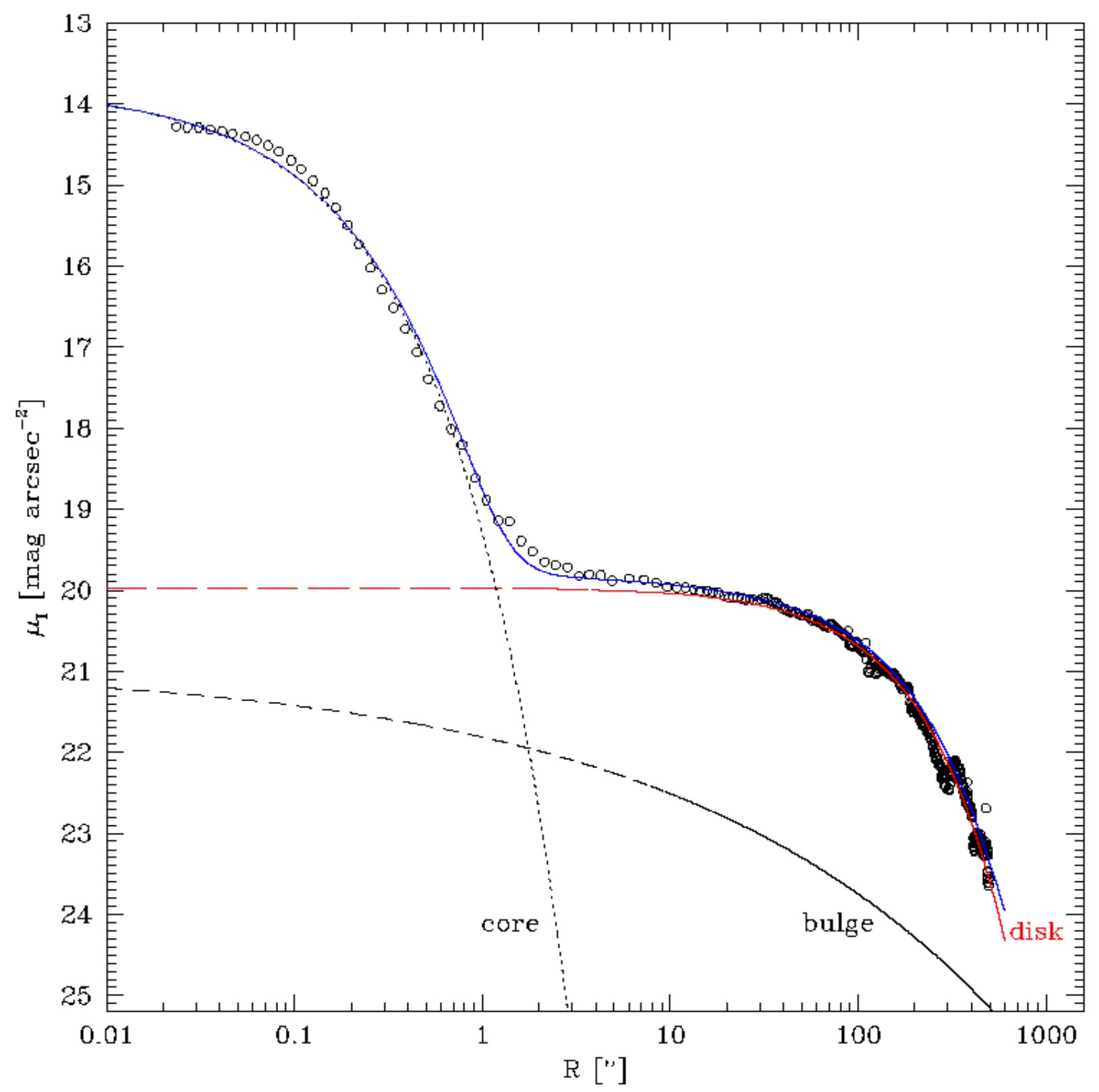

Figure 8 Profile decomposition of $I$-band surface brightness profile obtained in this study (for $r>5^{\prime \prime}$ ) combined with measurements from Böker et al. (2002; $\left.0 .{ }^{\prime \prime} 02<r<5^{\prime \prime}\right)$. The open circles are the observed data points, and the results of a three-component exponential disk (long-dashed line), $r^{1 / 4}$ bulge (short-dashed line) and Sersic core (dotted line) decomposition. The solid line is the combined result of the three model components. 
Böker T., Sarzi M., McLaughlin D. E., van der Marel R. P., Rix H.-W., Ho L. C., Shields J. C., 2004, AJ, 127,105

Butler D. J., Martínez-Delgado D., Brander W., 2004, AJ, 127, 1472

Carignan C., 1985, ApJS, 58, 107

Choi Y.-J., Park B.-G., Yoon T. S., Ann H. B., 1998, Jour. of the Korean Astron. Soc., 31, 141

Côté S., Freeman K. C., Carignan C., Quinn P. J., 1997, AJ, 114, 1313

de Vaucouleurs G., Page J., 1962, ApJ, 136, 107

de Vaucouleurs G., de Vaucouleurs A., Corwin H. G., Jr., Buta R. J., Paturel G., Fouqué P., 1991, Third Reference Catalogue of Bright Galaxies, New York: Springer-Verlag (RC3)

Elias J., 1994, NOAO Newsletter, No. 37, 1

Freedman W. L., et al., 2001, ApJ, 553, 47

Gebhardt K., et al., 2001, AJ, 122, 2469

Gebhardt K., Rich R. M., Ho L. C., 2002, ApJ, 578, L41

Ichikawa S., Okamura S., Watanabe M., Hamabe M., Aoki T., Kokaira K., 1987, Annals Tokyo Astron. Obs., 21,285

Karachentsev I. D., Grebel E. K., Sharina M. E., Dolphin A. E., Geisler D., Guhathakurta P., Hodge P. W., Karachentseva V. E., Sarajedini A., Seitzer P., 2003, A\&A, 404, 93

Kent S. M., 1983, ApJ, 266, 562

Kilkenny D., van Wyk F., Roberts G., Marang F., Coper D., 1998, MNRAS, 294, 93

Kim E., Lee M. G., \& Geisler D., 2000, MNRAS, 314, 307

Kim K. O., Ann H. B., 1990, Jour. of the Korean Astron. Soc., 22, 43

Kim S. C., Lee M. G., 1998, Jour. of the Korean Astron. Soc., 31, 51

Kim S. C., Sung H., Lee M. G., 2002, Jour. of the Korean Astron. Soc., 35, 9 (Paper I)

Kormendy J., McClure R. D., 1993, AJ, 105, 1793

Leinert Ch., Väisänen P., Mattila K., \& Lehtinen K., 1995, A\&AS, 112, 99

Menzies J., Marang F., Laing J. D., Coulson I. M., Engelbrecht C. A., 1991, MNRAS, 248, 642

Pietrzyński G., Gieren W., Fouqué P., Pont F., 2001, A\&A, 371, 497

Read A. M., Ponman T. J., Strickland D. K., 1997, MNRAS, 286, 626 
Richer H. B., Pritchet C. J., Crabtree D. R., 1985, ApJ, 298, 240

Rogstad D. H., Crutcher R. M., Chu K., 1979, ApJ, 229, 509

Sandage A., Bedke J., 1994, The Carnegie Atlas of Galaxies, Washington, D. C.: Carnegie Institution of Washington

Sandage A. R., Tammann G. A., 1981, A Revised Shapley-Ames Catalog of Bright Galaxies, Washington, D. C.: Carnegie Institution of Washington

Schlegel D., Finkbeiner D., Davis M., 1998, ApJ, 500, 525

Sersic J. L., 1968, Atlas de Galaxias Australes, Córdoba: Observatorio Astronómico

Soffner T., Méndez R. H., Jacoby G. H., Ciardullo R., Roth M. M., Kudritzki R. P., 1996, A\&A, 306, 9

Stephens A. W., Frogel J. A., 2002, AJ, 124, 2023

Sung H., Bessell M. S., 2000, PASA, 17, 244

van den Bergh S., 1999, ApJ, 517, L97

Whiting A. B., 1999, AJ, 117, 202 